\title{
SOLUTION OF A CONVERGENCE PROBLEM IN THE THEORY OF T-FRACTIONS
}

\author{
ROLF M. HOVSTAD
}

ABSTRACT. Let $f$ be a function, holomorphic in $|z|<R$, where $R>1$, normalized by $f(0)=1$, and satisfying a boundedness condition of the form $|f(z)-1|<K$. It is proved that a certain modification of the Thron continued fraction expansion of $f$ converges to $f$ uniformly on any $|z| \leq r<R$.

Introduction. In 1948 Thron [1] introduced continued fractions of the type

$$
1+d_{0} z+\frac{z_{0}}{1+d_{1} z}+\cdots+\frac{z}{1+d_{n} z}+\cdots \cdot
$$

They are called $T$-fractions. By definition, the $T$-fraction (1) converges for $z=z_{0}$ if

$$
\lim _{n \rightarrow \infty}\left(1+d_{0} z_{0}+\frac{z_{0}}{1+d_{1} z_{0}}+\cdots+\frac{z_{0}}{1+d_{n} z_{0}}\right)
$$

exists. T-fractions have been studied in [1], [2] and [3]. If we put

$$
1+d_{0} z+\frac{z}{1+d_{1} z}+\cdots+\frac{z}{1+d_{n} z}=\frac{A_{n}(z)}{B_{n}(z)},
$$

then $A_{n}$ and $B_{n}$ can be written as polynomials determined by the recurrence formulas

$$
\begin{gathered}
A_{-1}(z)=1, \quad A_{0}(z)=1+d_{0} z, \\
A_{n}(z)=\left(1+d_{n} z\right) A_{n-1}(z)+z A_{n-2}(z), \\
B_{-1}(z)=0, \quad B_{0}(z)=1, \\
B_{n}(z)=\left(1+d_{n} z\right) B_{n-1}(z)+z B_{n-2}(z), \quad n \geq 1 .
\end{gathered}
$$

Received by the editors December 21, 1972 and, in revised form, January 21, 1974.

AMS (MOS) subject classifications (1970). Primary 30A22, $40 \mathrm{~A} 15$.

Key words and phrases. Holomorphic function, $T$-fraction expansion, modified $T$-fraction expansion. 
Let $f_{0}$ be analytic for $z=0$ and $f_{0}(0)=1$. The sequence $\left\{f_{n}\right\}_{n \geq 0}$ is given by

$$
\begin{aligned}
f_{n}(z) & =1+\left(f_{n}^{\prime}(0)-1\right) z+z / f_{n+1}(z), \quad z \neq 0 \\
f_{n+1}(0) & =1
\end{aligned}
$$

and $f_{n}$ is analytic for $z=0$. If $d_{n}=f_{n}^{\prime}(0)-1$, the $T$-fraction (1) is called the $T$-fraction expansion of $f_{0}$.

In [4] H. Waadeland proved: There exists an $R_{0}>0$ such that $R>R_{0}$ implies the existence of a $K_{R}>0$ with the property: If $f_{0}$ is analytic for $|z|<R, f_{0}(0)=1$ and $\left|f_{0}(z)-1\right|<K_{R}$ in $|z|<R$, then the functions $f_{n}$ given by (2) are all analytic in $|z|<R$. Furthermore, $\lim _{n \rightarrow \infty} d_{n}=-1$. It is also shown that $R_{0} \geq 1$. The smallest value for $R_{0}$ given in [4] is $R_{0}=3 / 2$.

The quoted result on the sequence $\left\{f_{n}\right\}_{n} \geq 0$ is used in [4] to prove that the corresponding $T$-fraction expansion of $f_{0}$ converges to $f_{0}$ locally uniformly in $|z|<1$. It is pointed out in [5] that this $T$-fraction expansion never converges to $f_{0}$ in a larger disc.

In order to provide convergence in a larger disc a modification of the $T$ fraction expansion is studied in [5]. This modification is established by replacing the approximants $\left(1^{\prime}\right)$ by modified approximants

(3) $1+d_{0} z+\frac{z}{1+d_{1} z}+\cdots+\frac{z}{1+d_{n-1} z}+\frac{z}{1+\left(d_{n}+1\right) z}=\frac{A_{n}(z)+z A_{n-1}(z)}{B_{n}(z)+z B_{n-1}(z)}$.

Under the conditions on $f_{0}$ quoted above, the sequence of modified $T$. approximants converges to $f_{0}$ locally uniformly in the disc $|z|<2 R / 3$. The question is raised in [5] whether this result can be extended to a result on convergence in the whole disc $|z|<R$. It turns out that the crucial point in the solution of this question is the rate at which $f_{n}$ tends to 1 . The purpose of the present paper is to give an affirmative answer to this question.

A basic convergence property of the sequence $\left\{f_{n}\right\}_{n \geq 0}$. We proceed to prove the following basic result on the sequence $\left\{f_{n}\right\}_{n \geq 0}$, defined by (2).

Theorem 1. Let $R>1$ and choose $\gamma$ such that $1<y<R$. Then there exists a $K_{R}(\gamma)>0$ such that if $f_{0}$ is analytic in $|z|<R, f_{0}(0)=1$ and $|f(z)-1|<K_{R}(\gamma)$ in $|z|<R$, there exists a constant $C_{R}(\gamma)>0$ (independent of $n$ and $f_{0}$ ), such that 


$$
\left|f_{n}(z)-1\right| \leq C_{R}(\gamma)(y / R)^{n}
$$

for $|z|<R$ and $n \geq 0$.

The inequality (4) implies that all $f_{n}$ are analytic in $|z|<R$ and, by Schwarz' lemma, $\lim _{n \rightarrow \infty} d_{n}=-1$. Besides, $\lim _{n \rightarrow \infty} f_{n}(z)=1$ uniformly in $|z|<R$. It should be mentioned that (4) fails to hold in the case $R \leq 1$ (see [4]). The following proof originated from an analysis of [4, pp. 13-15].

Proof of (4). Let $f_{0}(z)=1+\sum_{q=1}^{\infty} a_{q} z^{q}$ for $|z|<R$. Using (2) we obtain for $n \geq 0$

$$
f_{n}(z)=\left(1+\sum_{q=1}^{\infty} a_{q}(n) z^{q}\right) /\left(1+\sum_{q=1}^{\infty} b_{q}(n) z^{q}\right)
$$

(for $z$ in a region $\Omega_{n}$ containing $z=0$ ), where

$$
\begin{aligned}
& a_{q}(n+1)=b_{q}(n), \quad a_{q}(0)=a_{q}, \quad b_{q}(0)=0, \\
& b_{q}(n+1)=a_{q+1}(n)-b_{q+1}(n)-b_{q}(n)\left(a_{1}(n)-b_{1}(n)-1\right) .
\end{aligned}
$$

If for $n \geq 0$ and $q \geq 1, c_{q}(n)=a_{q}(n)-b_{q}(n)$, the recurrence formulas (5) show that (for $n \geq 1, q \geq 1$ )

$$
c_{q}(n+1)=-c_{q+1}(n)-c_{1}(n) \sum_{k=1}^{n} c_{q}(k) .
$$

Using this and $c_{q}(0)=a_{q}, c_{q}(1)=-a_{q+1}$, we obtain for $q \geq 1$ and $n \geq 0$

$$
c_{q}(n)=(-1)^{n} a_{n+q}+s_{q}(n),
$$

where $s_{q}(n)$ is a linear combination of products (with at least 2 factors) of the numbers $a_{1}, a_{2}, a_{3}, \ldots$. Therefore we obtain for $n \geq 0$

$$
f_{n}^{\prime}(0)=c_{1}(n)=(-1)^{n} a_{n+1}+s_{1}(n) .
$$

Let $\mathcal{F}_{0}(K)$ be the family of functions $f_{0}$ such that $f_{0}$ is analytic in $|z|<$ $R, f_{0}(0)=1$ and $\left|f_{0}(z)-1\right|<K$ in $|z|<R(K>0)$. Furthermore, let $\mathcal{F}_{n}(K)$ be the family of functions $f_{n}$ determined by (2) from the functions $f_{0} \in \mathfrak{F}_{0}(K)$. We know that for $q \geq 1$

$$
\left|a_{q}\right| \leq K / R^{q} .
$$

For $n \geq 0$ the existence of $D_{n}(K)=\sup _{f_{n} \in \mathscr{F}_{n}(K)}\left|f_{n}^{\prime}(0)\right|$ is assured by (6), 
and (6) and (7) give

$$
\varlimsup_{K \rightarrow 0} \frac{D_{n}(K)}{K} \leq \frac{1}{R^{n+1}}
$$

From (2) we get

$$
f_{n+1}(z)-1=-\frac{\left(f_{n}(z)-1\right) / z-f_{n}^{\prime}(0)}{1+\left(f_{n}(z)-1\right) / z-f_{n}^{\prime}(0)}
$$

(which in general is meromorphic in $|z|<R$ ).

Let $m \geq 1$ be an integer such that $1<\sqrt[m]{m+1}<\gamma<R$. Using (9) we see that there exists a constant $H_{1}>0$ such that if $0<K<H_{1}$ and $0 \leq$ $n \leq m$, the functions in $\mathcal{F}_{n}(K)$ are analytic in $|z|<R$ and

$$
F_{n}(K)=\operatorname{Sup}_{|z|<R ; f_{n} \in \mathcal{F}_{n}(K)}\left|f_{n}(z)-1\right|
$$

exists. (9) and (8) then give $(0 \leq n \leq m)$

$$
\varlimsup_{K \rightarrow 0} \frac{F_{n}(K)}{K} \leq \frac{n+1}{R^{n}} .
$$

Thus, if $\theta \in\left\langle(m+1) / R^{m}, 1\right\rangle$ there exists an $H_{2} \in\left\langle 0, H_{1}\right\rangle$ such that $0<K$ $\leq H_{2}$ and $f_{m} \in \mathcal{F}_{m}(K)$ implies

$$
\left|f_{m}(z)-1\right|<\theta K
$$

in $|z|<R$. Because of (12) and (2) we have $\mathcal{F}_{p m+q}(K) \subseteq \mathcal{F}_{q}(K)$ for $p \geq 0$, $0 \leq q \leq m-1$ and $0<K \leq H_{2}$. Thus the functions in $\mathcal{F}_{n}(K)$ are analytic in $|z|<R$ for $n \geq 0\left(0<K \leq H_{2}\right)$.

Further, according to (12), we have $\mathcal{F}_{p m+q}(K) \subseteq \mathcal{F}_{q}\left(\theta^{p} K\right)$ for $0<K \leq$ $H_{2}, p \geq 0$ and $0 \leq q \leq m-1$. Therefore the inequality

$$
F_{q}\left(\theta^{p} K\right) \geq \underset{|z|<R_{;} f_{n} \in \mathcal{F}_{n}(K)}{ }\left|f_{n}(z)-1\right|
$$

holds for $0<K \leq H_{2}, n=p m+q, p \geq 0$ and $0 \leq q \leq m-1$. Because of (10) and (11) the existence of the positive number

$$
M=\operatorname{Max}_{0 \leq q \leq m-1} \operatorname{Sup}_{0<K \leq H_{2}} \frac{F_{q}(K)}{K}
$$

is assured (observe that $F_{q}\left(K_{1}\right) \leq F_{q}\left(K_{2}\right)$ for $\left.0<K_{1} \leq K_{2}\right)$. Therefore $F_{q}(K)$ 
$\leq M \cdot K$ for $0<K \leq H_{2}$ and $0 \leq q \leq m-1$. Thus (for $n=p m+q, p \geq 0$ and $0 \leq q \leq$ $m-1)$

$$
F_{q}\left(\theta^{p} H_{2}\right) \leq M \cdot H_{2} \cdot \theta^{p} \leq M H_{2} / \theta \cdot \theta^{n / m}=C \cdot \theta^{n / m} .
$$

(13) now gives for $n=p m+q, p \geq 0$ and $0 \leq q \leq m-1$

$$
\operatorname{Sup}_{|z|<R ; f_{n} \in \mathcal{F}_{n}\left(H_{2}\right)}\left|f_{n}(z)-1\right| \leq C \cdot \theta^{n / m} .
$$

As a result we see that if $f_{0}$ is analytic in $|z|<R, f_{0}(0)=1,\left|f_{0}(z)-1\right|<$ $H_{2}$ and $\left\{f_{n}\right\}_{n \geq 0}$ is determined recursively by (2) from $f_{0}$, then $\left|f_{n}(z)-1\right| \leq$ $C \cdot \theta^{n / m}$ in $|z|<R$. Choosing $\theta=(\gamma / R)^{m}$ we see that (4) is proved (with $C_{R}(\gamma)=C$ and $\left.K_{R}(\gamma)=H_{2}\right)$.

Application to the modified $T$-fraction expansion. Using (4) and following $\mathrm{H}$. Waadeland [5, pp. 6-10], we are able to conclude the result:

Theorem 2. Let $R>1$ and $0<r<R$. Then there exists a $K_{r}>0$ such that if $f_{0}$ is analytic in $|z|<R, f_{0}(0)=1$ and $\left|f_{0}(z)-1\right|<K_{r}$ in $|z|<R$, then the modified $T$-fraction expansion converges to $f_{0}$ uniformly in $|z| \leq$ $\leq r<R$.

Proof. The difference between $f_{0}(z)$ and the $(n-1)$ th modified approximant may be written

$$
f_{0}(z)-\frac{A_{n-1}(z)+z A_{n-2}(z)}{B_{n-1}(z)+z B_{n-2}(z)}=\frac{(-1)^{n-1} z^{n}(1-f(z))}{H_{n}(z)\left(H_{n}(z)+\left(1-f_{n}(z)\right) B_{n-1}(z)\right)}
$$

where $H_{n}(z)=\Pi_{k=1}^{n} f_{k}(z)$. From (4) and (2) we conclude that the infinite product $\prod_{k=1}^{\infty} f_{k}(z)$ converges uniformly on $|z|<R$ and $\Pi_{k=1}^{\infty} f_{k}(z) \neq 0$ on $|z|<R$.

Let $0<r<R$ and choose $\gamma$ such that $r<R / y<R$ and $1<\gamma<R$. Denote $K_{r}=K_{R}(y)\left(K_{R}(y)\right.$ in Theorem 1$)$. We then have from (4)

for $|z| \leq r$.

$$
\left|z^{n}\left(1-f_{n}(z)\right)\right| \leq C_{R}(\gamma)(r y / R)^{n}
$$

Further we use

$$
\begin{aligned}
B_{n}(z)-1=- & z\left(B_{n-1}(z)-1\right) \\
& +z \sum_{k=1}^{n}\left(1+d_{k}\right)\left(B_{k-1}(z)-1\right)-z+z \sum_{k=1}^{n}\left(1+d_{k}\right) .
\end{aligned}
$$


This gives for $|z| \leq r$ (using (4))

$$
\begin{aligned}
\left|B_{n}(z)-1\right| \leq r\left|B_{n-1}(z)-1\right|+r+r \sum_{k=1}^{n}\left|1+d_{k}\right| \\
\quad+r \sum_{k=1}^{n}\left|1+d_{k}\right| \cdot\left|B_{k-1}(z)-1\right| \\
\leq r\left|B_{n-1}(z)-1\right|+r+\frac{r y C_{R}(\gamma)}{R(R-\gamma)}+\frac{r C_{R}(\gamma)}{R} \sum_{k=1}^{n}\left(\frac{\gamma}{R}\right)^{k}\left|B_{k-1}(z)-1\right| .
\end{aligned}
$$

Let $\alpha>1$ be a number such that $r<\alpha<R / \gamma$. Next we choose $n$ and a constant $G \geq 1$ such that $\left(1+2 \gamma C_{R}(\gamma) / R(R-\alpha \cdot \gamma)\right)\left(1 / \alpha^{n}\right) \leq 1 / r-1 / \alpha$ and $\left|B_{k}(z)-1\right| \leq G \alpha^{k}$ for $0 \leq k \leq n-1$. We are now able to conclude that

$$
\begin{aligned}
\left|B_{n}(z)-1\right| & \leq r G \alpha^{n-1}+r+\frac{r y C_{R}(\gamma)}{R(R-\gamma)}+\frac{r y C_{R}(\gamma) G}{R(R-\alpha \gamma)} \\
& \leq G r\left(\alpha^{n-1}+1+\frac{2 \gamma C_{R}(\gamma)}{R(R-\alpha \gamma)}\right) \leq G r\left(\alpha^{n-1}+\alpha^{n}\left(\frac{1}{r}-\frac{1}{\alpha}\right)\right)=G \alpha^{n} .
\end{aligned}
$$

By induction we can now easily prove that $\left|B_{k}(z)-1\right| \leq G a^{k}$ for all $k \geq 0$. Thus we have for $|z| \leq r$

$$
\left|\left(1-f_{n}(z)\right) B_{n-1}(z)\right| \leq C_{R}(\gamma)(\gamma / R)^{n}\left(1+G \alpha^{n-1}\right) \leq 2 G C_{R}(\gamma)(\alpha \gamma / R)^{n} .
$$

From the results obtained we are now able to conclude that

$$
\lim _{n \rightarrow \infty} \frac{A_{n-1}(z)+z A_{n-2}(z)}{B_{n-1}(z)+z B_{n-2}(z)}=f_{0}(z)
$$

uniformly on $|z| \leq r$ and the theorem is proved.

We will now apply Theorem 2 to

Theorem 3. Let $R>1$. Let $K_{R}>0$ be a number such that $f_{0}$ analytic in $|z|<R, f_{0}(0)=1$ and $\left|f_{0}(z)-1\right|<K_{R}$ in $|z|<R$ imply that $\lim _{n \rightarrow \infty} f_{n}(z)=$ 1 uniformly in $|z|<R$. Then the modified $T$-fraction expansion converges locally uniformly to $f_{0}$ in $|z|<R$.

Proof. Let $0<r<R$. From Theorem 2 we pick out the number $K_{r}$. Now we choose $m$ such that $\left|f_{m}(z)-1\right|<K_{r}$ for $|z|<R$. From Theorem 2 we know that the modified $T$-fraction expansion of $f_{m}$ converges uniformly 
to $f_{m}$ on $|z| \leq r$. Because of the recursive properties of $\left\{f_{n}\right\}_{n \geq 0}$ and the sequence of modified $T$-fractions we obtain our theorem.

It is clear that the existence of a $K_{R}$ in Theorem 3 is assured by Theorem 1. To present explicitly a $K_{R}$ which works requires of course some direct calculation. As an example we will give a $K_{R}$ for the case $R>2$ based upon the proof of Theorem 3.1 in [4].

Example. Let $R\rangle$ 2. Then all numbers in $\langle 0, R / 2-1\rangle$ can be used as a $K_{R}$ in Theorem 3 .

Proof. If $0<K_{R}<R / 2-1$, then $0<2 /\left(R-2 K_{R}\right)<1$, and it follows easily from (2) that for $n \geq 0$

$$
\left|f_{n}(z)-1\right|<\left(2 i\left(R-2 K_{R}\right)\right)^{n} K_{R}
$$

in $|z|<R$.

The author wishes to express his thanks to W. Thron and H. Waadeland for useful criticism related to the part of the paper containing the application to the modified $T$-fraction expansion. Also, the referee is thanked for reading the manuscript and all his valuable suggestions.

\section{REFERENCES}

1. W. J. Thron, Some properties of continued fractions $1+d_{0} z+$ $K\left(z /\left(1+d_{n} z\right)\right)$, Bull. Amer. Math. Soc. 54 (1948), 206-218. MR 9, 508.

2. W. B. Jones, Contributions to the theory of Thron continued fractions, Ph.D. Thesis, Vanderbilt University, Nashville, Tenn., 1963.

3. W. B. Jones and W. J. Thron, Further properties of T-fractions, Math. Ann. 166 (1966), 106-118. MR 34 \#319.

4. H. Waadeland, On T-fractions of functions, holomorphic and bounded in a circular disc, Norske Vid. Selsk. Skr. (Trondheim) No. 8 (1964), 19 pp.

MR $31 \# 1364$.

5. A convergence property of certain T-fraction expansions, Norske Vid. Selsk. Skr. (Trondheim) No. 9 (1966), 22 pp. $\quad$ MR 37 \#1568. NOR WAY

DEPART MENT OF MATHEMATICS, UNIVERSITY OF TRONDHEIM, TRONDHEIM, 